\title{
Evaluation of semi-quantitative compared to quantitative cultures of tracheal aspirates for the yield of culturable respiratory pathogens - a cross-sectional study
}

Salima Rattani, Joveria Farooqi, Ghazala Jabeen, Saeeda Chandio, Qaiser Kash, Aijaz Khan and Kauser Jabeen* (1)

\begin{abstract}
Background: Diagnosis of lower respiratory tract infections (LRTI) depends on the presence of clinical, radiological and microbiological findings. Endotracheal suction aspirate (ETSA) is the commonest respiratory sample sent for culture from intubated patients. Very few studies have compared quantitative and semi-quantitative processing of ETSA cultures for LRTI diagnosis. We determined the diagnostic accuracy of quantitative and semi-quantitative ETSA culture for LRTI diagnosis, agreement between the quantitative and semi quantitative culture techniques and the yield of respiratory pathogens with both methods.

Methods: This was a cross-sectional study conducted at the Aga Khan University clinical laboratory, Karachi, Pakistan. One hundred and seventy-eight ETSA samples sent for routine bacteriological cultures were processed quantitatively as part of regular specimen processing method and semi-quantitatively. Sensitivity, specificity, positive predictive value (PPV), negative predictive value (NPV) and diagnostic accuracy was calculated for both methods using clinical diagnosis of pneumonia as reference standard. Agreement between the quantitative and semi quantitative methods was assessed via the kappa statistic test. Pathogen yield between the two methods was compared using Pearson's chi-square test.
\end{abstract}

Results: The quantitative and semi-quantitative methods yielded pathogens in 81 (45.5\%) and 85 (47.8\%) cases respectively. There was complete concordance of both techniques in 155 (87.1\%) ETSA samples. No growth was observed in 45 (25.3\%) ETSA specimens with quantitative culture and 37 (20.8\%) cases by semi-quantitative culture. The diagnostic accuracy of both techniques were comparable; $64.6 \%$ for quantitative and $64.0 \%$ for semi-quantitative culture. The kappa agreement was found to be 0.84 ( $95 \% \mathrm{Cl}, 0.77-0.91)$ representing almost perfect agreement between the two methods. Although semiquantitative cultures yielded more pathogens (47.8\%) as compared to quantitative ETSA cultures (45.5\%), the difference was only 2.3\%. However, this difference achieved statistical (chi-square $p$-value $<0.001$ ) favoring semi-quantitative culture methods over quantitative culture techniques for processing ETSA.

(Continued on next page)

\footnotetext{
* Correspondence: kausar.jabeen@aku.edu

Department of Pathology \& Laboratory Medicine, The Aga Khan University,

Stadium Road, Karachi 74800, Pakistan
} 
(Continued from previous page)

Conclusion: In conclusion, there is a strong agreement between the performances of both methods of processing ETSA cultures in terms of accuracy of LRTI diagnosis. Semi-quantitative cultures of ETSA yielded more pathogens as compared to quantitative cultures. Although both techniques were comparable, we recommend processing of ETSA using semiquantitative technique due to its ease and reduced processing time.

Keywords: Tracheal aspirate, Endotracheal suction aspirate, Quantitative culture, Semi-quantitative culture, Lower respiratory tract infections, Pneumonia, Respiratory pathogens, Pakistan

\section{Background}

Lower respiratory tract infections (LRTI) are a cause of increased morbidity and mortality in critically ill patients [1]. It is one of the leading infective causes of intensive care units (ICU) admissions [2, 3]. Its diagnosis is based on the presence of clinical findings along with radiological and microbiological findings [4]. Types of respiratory samples recommended for culture include sputum, endotracheal suction aspirates (ETSA), bronchoalveolar lavage (BAL), protected brush specimens (PBS). Postphysiotherapy ETSA is a much easier sample to collect in intubated patients compared to an invasive BAL sample. These can be obtained simply and cost effectively with less side-effects as compared to BAL and PBS [5]. Thus the commonest respiratory sample from intubated patients with suspected pneumonia or lower respiratory tract infection (LRTI) sent for microbiological analysis is ETSA [3]. For a proper microbiological diagnosis, a true representative sample is necessary as the respiratory tract, endotracheal tubes and tracheostomies are commonly colonized with normal flora [6]. Usual cultivable pathogens causing LRTI are Streptococcus pneumoniae, Haemophilus influenzae, Moraxella catarrhalis, Staphylococcus aureus, Klebsiella pneumoniae and Pseudomonas aeruginosa in patients with CAP [7] while Acinetobacter species, $P$. aeruginosa, S. aureus and $K$. pneumoniae are more prevalent in HAP [8]. Recent guidelines published by the Infectious Disease Society of America (IDSA) in 2016 recommend use of noninvasive semiquantitative cultures in these patients [9]. However, the international European Respiratory Society (ERS), European Society of Intensive Care Medicine (ESICM), the European Society of Clinical Microbiology and Infectious Diseases (ESCMID) and the Latin American Thoracic Association (ALAT) guidelines for the management of hospital-acquired pneumonia and ventilator-associated pneumonia recommend obtaining distal quantitative samples (prior to any antibiotic treatment) in order to reduce antibiotic exposure in stable patients with suspected ventilator associated pneumonia (VAP) and to improve the accuracy of the results; and a lower respiratory tract sample (distal quantitative including BAL and PSB or proximal quantitative or qualitative culture including ETSA) to focus and narrow the initial empiric antibiotic therapy [10]. There have been multiple studies comparing the results of quantitative BAL cultures with ETSA cultures with similar results $[9,11]$.Very few studies compare quantitative and semi-quantitative processing of ETSA cultures for pathogen yield. We hypothesized that the performance of ETSA culture using quantitative or semi-quantitative technique for LRTI diagnosis is similar.

Processing of cultures quantitatively is more time consuming and costly as compared to semi-quantitative processing of ETSA. The objective of our study was to determine the diagnostic accuracy of quantitative and semi-quantitative ETSA culture for LRTI diagnosis, agreement between the quantitative and semi quantitative culture techniques and the yield of respiratory pathogens with both methods.

\section{Methods}

This was a cross-sectional study conducted at the microbiology section of clinical laboratory of Aga Khan University Hospital - a tertiary care hospital in Karachi, Pakistan as a part of a quality improvement project. ETSA samples received at the AKUH clinical Laboratories for routine bacteriological cultures, selected by systematic sampling, were included in the study. First five ETSA samples received in the microbiology section after 8 am were processed in parallel by the two methods daily Monday through Thursday, from June to September 2017 and then from October to November 2019. We planned to exclude those ETSA samples which were too small in quantity to be processed by both methods. However, we did not encounter any insufficient samples.

Samples were processed both quantitatively as part of regular specimen processing method for ETSA and semi-quantitatively as well. For quantitative cultures, ETSA were digested using an equal amount of sputasol (dithiothreitol) and mixed on a vortex mixer (1:2 dilution). $100 \mu \mathrm{l}(0.1 \mathrm{ml})$ of the digested specimen was diluted into $9.9 \mathrm{ml}$ of Ringers' solution (1:200 of the original sample). $10 \mu \mathrm{l}(0.01 \mathrm{ml})$ of the diluted sample was then inoculated on Blood Colistin Nalidixic Acid Agar (BCNA), Chocolate Agar (CHOC) (both incubated in $5 \% \mathrm{CO} 2$ at $37{ }^{\circ} \mathrm{C}$ ) and MacConkey Agar (MAC) (incubated in ambient environment at $37{ }^{\circ} \mathrm{C}$ ) and streaked in quadrants. The quantitative cultures were considered significant at a count of $\geq 10^{5}$ colony forming units $/ \mathrm{ml}$ of pathogenic organisms, i.e. $\geq 5$ colonies of the same type 
of organism on a non-selective medium (CHOC agar). For semi-quantitative cultures, samples were examined for purulence and a loopful of the most purulent part was inoculated on $\mathrm{CHOC}, \mathrm{BCNA}$ and MAC agars and streaked in quadrants. Results were considered significant if there was moderate to heavy growth (colonies growing up to secondary or tertiary streaks) of organisms known to cause lower respiratory tract infection. Final yield was determined after two days of incubation.

Lower Respiratory Tract Infections (LRTI) include bronchitis and bronchiolitis - commonly caused by viruses and atypical bacteria; community-acquired pneumonia (CAP); hospital-acquired pneumonia (HAP) and ventilator-associated pneumonia (VAP); infections of the pleural space; bronchopulmonary infections in patients with cystic fibrosis; and pneumonia in the immunocompromised host [12]. Community Acquired Pneumonia was defined as a new lung infiltrate plus clinical evidence that the infiltrate was of an infectious origin, which included the new onset of fever, purulent sputum, leukocytosis, and decline in oxygenation, excluding hospital acquired pneumonia. Hospital Acquired Pneumonia was defined as a pneumonia not incubating at the time of hospital admission and occurring $48 \mathrm{~h}$ or more after admission. Ventilator Associated Pneumonia was defined as pneumonia in mechanically ventilated patients that developed later than or at $48 \mathrm{~h}$ after the patient was placed on mechanical ventilation [9].

Concordance between culture methods was expressed as a percentage and was determined by comparing the quantitative and semi-quantitative culture results of ETSA. The results were considered to be completely concordant if both culture methods yielded either no growth or had identical growth of pathogens. The growth of pathogens by ETSA below the cutoff value of $\geq 10^{5}$ colony forming units $/ \mathrm{ml}$ or of oral flora such as alpha hemolytic streptococci or yeast was considered as no significant bacterial pathogen isolated. Yield of no organisms on culture after two days of incubation was considered as no growth. The results were considered to be completely discordant when growth of pathogens occurred via one method and not by the other, or when pathogens grew via both methods but the isolates identified were different.

A minimum sample size of 173 ETSA samples to be processed by both quantitative and semi-quantitative culture techniques was calculated to determine with 95\% confidence whether pathogen yield is comparable between the two methods.

Statistical analysis was performed using SPSS version 23. Means and standard deviation were used to compute continuous variables like age. Frequency and percentage were used to analyze qualitative variables like gender. Pathogen yield between the two methods was compared using Pearson's chi-square test. Agreement between the quantitative and semi quantitative microbiological results obtained with ETSA was assessed via the kappa statistic test and interpreted as follows: values $\leq 0$ as no agreement, and $0.01-0.20$ as none to slight, $0.21-0.40$ as fair, $0.41-0.60$ as moderate, $0.61-0.80$ as substantial, and $0.81-1.00$ as almost perfect agreement [13]. Sensitivity, specificity, positive predictive value (PPV), negative predictive value (NPV), postive likelihood ratio (PLR), negative likelihood ratio (NLR) and diagnostic accuracy was calculated for both methods using clinical diagnosis of LRTI as reference standard.

\section{Results}

One hundred and seventy-eight ETSA samples were processed by both quantitative and semi-quantitative culture techniques. These included samples from patients with HAP including VAP and VAE along with CAP, aspiration pneumonia and bronchopulmonary disease. The general characteristics for the patients are shown in Table 1.

The pathogen yield between the two methods was found to be significantly different (chi-square $p$-value $<0.001$ ) with semi-quantitative cultures yielding more pathogens $(47.8 \%)$ as compared to quantitative ETSA cultures (45.5\%). Even though, the difference was only $2.3 \%$, this difference achieved statistical significance favoring semiquantitative culture methods over quantitative culture techniques for processing ETSA. The quantitative method and semi-quantitative method revealed no growth in 45 (25.3\%) and $37(20.8 \%)$ cases, no significant bacterial pathogen was isolated in $52(29.2 \%)$ and $56(31.5 \%)$ cases while pathogens were isolated in 81 (45.5\%) and 85 (47.8\%) cases respectively, as shown in Fig. 1.

The most common pathogens isolated included P. aeruginosa, S. aureus, Acinetobacter species, K. pneumoniae. More than one pathogen was isolated in 20 cases. Table 2 shows the details of pathogens isolated.

Out of the 178 samples that were processed both quantitatively and semi-quantitatively, there was complete concordance between 155 (87.1\%) samples and discordant results were found in 23 (12.9\%) samples. Out of these 23 discordant findings, 6 were due to the presence or absence of oral flora on either culture, 4 were due to the presence of additional organism in semi-quantitative in an insignificant count, 3 were due to an additional organism present on the quantitative as compared to semi-quantitative while 10 were due to presence of additional pathogenic organisms on semi-quantitative in comparison with quantitative. The kappa agreement between the two methods was found to be 0.84 (95\% CI, 0.77-0.91) representing strong correlation, if they were compared according to categories of "no significant bacteria isolated", "no growth" or "pathogen isolated" while it was 0.93 (95\% CI, 0.880.99) representing almost perfect correlation, if 
Table 1 General characteristics of study population including gender, age distribution, clinical diagnosis and organisms isolated

\begin{tabular}{|c|c|}
\hline Number of samples & 178 \\
\hline \multicolumn{2}{|l|}{ Gender } \\
\hline Male & 122 \\
\hline Female & 56 \\
\hline \multicolumn{2}{|l|}{ Age } \\
\hline$\leq 1$ month & 2 \\
\hline$\leq 1$ year & 11 \\
\hline$>1$ year to 5 years & 06 \\
\hline $6-17$ years & 2 \\
\hline $18-64$ years & 104 \\
\hline$\geq 65$ years & 53 \\
\hline Lower respiratory tract infection (LRTI) & 96 \\
\hline Hospital Acquired pneumonia (HAP) & 63 \\
\hline Ventilator associated pneumonia (VAP) & 48 \\
\hline Ventilator associated event (including Tracheitis) & 15 \\
\hline Community acquired pneumonia (CAP) & 4 \\
\hline Aspiration pneumonia & 11 \\
\hline Bronchopulmonary Disease (COPD, Asthma, CF) & 7 \\
\hline Others & 11 \\
\hline \multicolumn{2}{|l|}{ Organisms } \\
\hline Staphylococcus aureus & 18 \\
\hline Pseudomonas aeruginosa & 19 \\
\hline Acinetobacter species & 22 \\
\hline Klebsiella pneumoniae & 20 \\
\hline Other organisms & 20 \\
\hline \multicolumn{2}{|l|}{ Gram negative bacilli } \\
\hline Escherichia coli & 7 \\
\hline Stenotrophomonas maltophilia & 5 \\
\hline Serratia species & 1 \\
\hline \multicolumn{2}{|l|}{ CAP pathogens } \\
\hline Streptococcus pneumoniae & 2 \\
\hline Haemophilus influenzae & 1 \\
\hline Molds & 3 \\
\hline
\end{tabular}

comparison was done on the basis of "pathogen isolated" or "no pathogen isolated". Table 3 shows the sensitivity, specificity, positive predictive value, negative predictive value and diagnostic accuracy for both methods compared to clinical diagnosis of lower respiratory tract infection.

\section{Discussion}

The diagnosis of pneumonia in patients with lower respiratory tract infections (LRTI) is challenging and involves clinical, radiological and microbiological criteria. To fulfil the microbiological criteria, ETSA are the easiest and rapidly obtainable noninvasive specimens with semiquantitative results having highest sensitivity but least specificity [9, 14-17]. Less resources and expertise is needed for semi-quantitative processing which can be done rapidly compared to quantitative processing of ETSA [9].

The present study compares culture results for the two methods of processing ETSA suggesting that the results of these cultures processed using quantitative or semi-quantitative methods are comparable, and there is strong agreement between the results of the two methods. Our results are similar to study by Hoshimoto et al. [1], who found a significant correlation between the two culture techniques.

An interesting finding in our study was that the most common pathogen was Acinetobacter species, followed by Klebsiella pneumonia, Pseudomonas aeruginosa and Staphylococcus aureus showing an abundance of pathogens that commonly cause HAP as most of these were isolated from ETSA samples obtained from admitted patients. Studies from our center have shown atypical pathogens are more common in the etiology of pneumonia [18], however, recently, amongst cultivable organisms, Staphylococcus aureus was the most common pathogen causing pneumonia, with $S$. pneumoniae and $P$. aeruginosa being close seconds [19]. Another study looking at bacterial etiology of pneumonia in immunocompetent hospitalized patients showed Pseudomonas aeruginosa to be the most common causative agent in Pakistan [20]. Only 2 samples were positive for Streptococcus pneumoniae, historically considered the most common etiologic agent of CAP worldwide. Patients in Pakistan receive empiric antibiotics in outpatient and inpatient settings without prior microbiologic confirmation of etiologic agents which can lead to false negative cultures, and then later superimposed hospital acquired infections. These may be factors that influenced our low culture rates and a spectrum suggestive of nosocomial etiology [18].

The sensitivity and specificity of semi-quantitative culture for diagnosis of LRTI in our study was $60.4 \%$ and $68.3 \%$ respectively which is comparable to the study conducted by Fujitani et al. who showed a sensitivity and specificity of semi-quantitative endotracheal aspirate culture to be $65.4 \%$ and $56.1 \%$ respectively [11]. Multiple studies comparing invasive and non-invasive lower respiratory tract cultures for diagnosis of VAP have been done which show no difference in 28-day mortality, overall mortality, length of ICU stay, duration of mechanical ventilation, or antibiotic changes $[5,6,8,9]$. The 2016 IDSA guidelines [9] showed a summary of performance characteristics ETSA for microbiological diagnosis of pneumonia. They stated that sensitivity and specificity was $75 \%$ (95\% CI, 58-88) and 47\% (95\% CI, 29-65) respectively for ETSA with any amount of growth; positive predictive values ranged from 61\% (95\% 


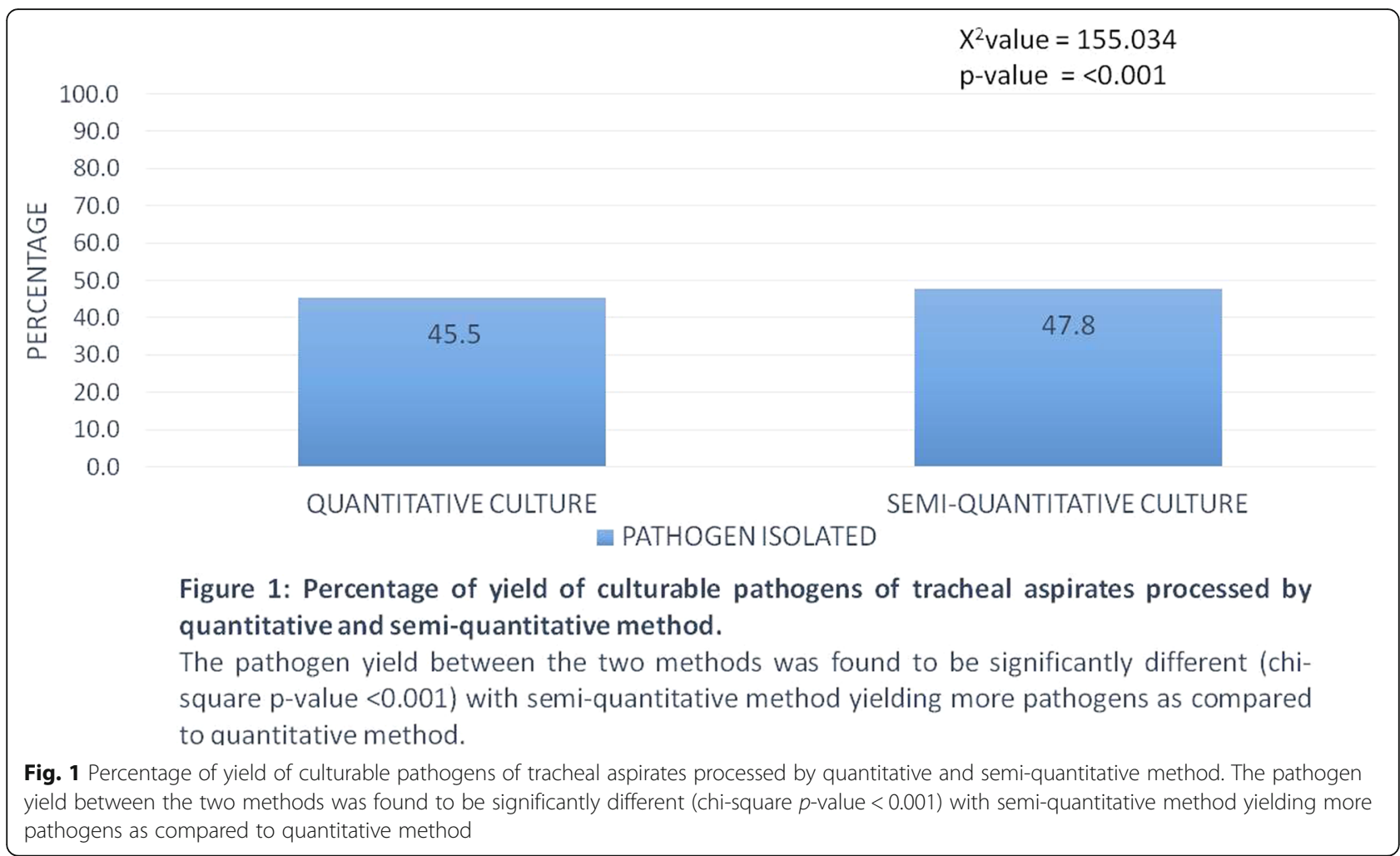

CI, 45-76) for ETSA with any amount of growth to $81 \%$ (95\% CI, 67\%-91\%) for ETSA with $\geq 10^{5} \mathrm{CFU} / \mathrm{ml}$. On the basis of these results they recommended noninvasive sampling with semi-quantitative cultures to diagnose VAP, rather than invasive or noninvasive sampling with quantitative cultures which is supported by our study as well.

Based on these recommendations and with our findings supporting the equivalence of the two techniques, laboratories could switch to semi-quantitative processing technique. An additional benefit of semi-quantitative processing for ETSA is reduction of technologist time, reagent consumption and chances for laboratory contamination due to less sample manipulation. This will in turn decrease the overall test cost for ETSA cultures.

There are several important limitations to this study that deserve attention. The sample size was relatively small. This was a single center study and most of the patients were hospitalized which explains the abundance of pathogens commonly associated with HAP, hence it may not be representative of other institutions. However, given that tracheal secretions are often submitted to the microbiology

Table 2 Percentage of isolation of different pathogens by quantitative and semi-quantitative ETSA cultures and by both methods

\begin{tabular}{llll}
\hline Organism Isolated & $\begin{array}{l}\text { By Quantitative Method } \\
\mathbf{n}(\%)\end{array}$ & $\begin{array}{l}\text { By Semi-Quantitative Method } \\
\mathbf{n}(\%)\end{array}$ & $\begin{array}{l}\text { By Both Method } \\
\mathbf{n}(\%)\end{array}$ \\
\hline Acinetobacter species & $22(23.2)$ & $23(23.7)$ & $22(23.7)$ \\
Klebsiella pneumoniae & $18(19.0)$ & $20(21.0)$ & $18(19.4)$ \\
Pseudomonas aeruginosa & $18(19.0)$ & $18(18.6)$ & $17(18.3)$ \\
Staphylococcus aureus & $18(19.0)$ & $17(17.5)$ & $17(18.3)$ \\
Escherichia coli & $7(7.4)$ & $7(7.2)$ & $7(7.5)$ \\
Stenotrophomonas maltophilia & $5(5.3)$ & $5(5.2)$ & $5(5.4)$ \\
Serratia species & $1(1.1)$ & $1(1.0)$ & $1(1.1)$ \\
Streptococcus pneumoniae & $2(2.1)$ & $2(2.1)$ & $2(2.2)$ \\
Haemophilus influenzae & $1(1.1)$ & $1(1.0)$ & $1(1.1)$ \\
Molds & $3(3.2)$ & $3(3.1)$ & $3(3.2)$ \\
Total organisms isolated & 95 & 97 & 93 \\
\hline
\end{tabular}


Table 3 Performance of quantitative culture and semi-quantitative culture techniques to detect pathogen for microbiological diagnosis compared to the clinical diagnosis of lower respiratory tract infection

\begin{tabular}{|c|c|c|c|c|}
\hline \multirow[t]{2}{*}{ Statistic } & \multicolumn{2}{|c|}{ Quantitative Culture } & \multicolumn{2}{|c|}{ Semi Quantitative Culture } \\
\hline & Value & $95 \% \mathrm{Cl}$ & Value & $95 \% \mathrm{Cl}$ \\
\hline Sensitivity & $59.4 \%$ & $48.9 \%$ to $69.3 \%$ & $60.4 \%$ & $49.9 \%$ to $70.3 \%$ \\
\hline Specificity & $70.9 \%$ & $59.6 \%$ to $80.6 \%$ & $68.4 \%$ & $56.9 \%$ to $78.4 \%$ \\
\hline Positive Likelihood Ratio & 2.04 & 1.39 to 2.99 & 1.91 & 1.33 to 2.74 \\
\hline Negative Likelihood Ratio & 0.57 & 0.43 to 0.76 & 0.58 & 0.43 to 0.77 \\
\hline Positive Predictive Value & $71.3 \%$ & $62.9 \%$ to $78.4 \%$ & $69.9 \%$ & $61.8 \%$ to $76.9 \%$ \\
\hline Negative Predictive Value & $59.0 \%$ & $52.0 \%$ to $65.5 \%$ & $58.7 \%$ & $51.6 \%$ to $65.5 \%$ \\
\hline Diagnostic Accuracy & $64.6 \%$ & $57.0 \%$ to $71.6 \%$ & $64.0 \%$ & $56.4 \%$ to $71.1 \%$ \\
\hline
\end{tabular}

laboratory as part of an empiric work-up for fever in a hospitalized patient, it is suspected that the data here are generally representative of institutions where ETSA culture is routinely performed.

\section{Conclusion}

In conclusion, there is a strong agreement between the performances of both methods of processing ETSA cultures in terms of accuracy of diagnosis of LRTI. Semiquantitative cultures of ETSA yielded more pathogens as compared to quantitative cultures. Although both techniques were comparable, we recommend processing of ETSA using semi-quantitative technique due to its ease and reduced processing time.

\section{Abbreviations}

CAP: Community acquired pneumonia; HAP: Hospital acquired pneumonia; LRTI: Lower respiratory tract infection; VAP: Ventilator associated pneumonia; ETSA: Endotracheal suction aspirates; BAL: Bronchoalveolar lavage; PBS: Protected brush specimens; ICU: Intensive care unit; IDSA: Infectious Disease Society of America; ERS: European Respiratory Society; ESIC M: European Society of Intensive Care Medicine; ESCMID: European Society of Clinical Microbiology Infectious Diseases; ALAT: Latin American Thoracic Association; BCNA: Blood Colistin Nalidixic Acid Agar; CHOC: Chocolate Agar; MAC: MacConkey Agar; PPV: Positive predictive value; NPV: Negative predictive value

\section{Acknowledgements}

We would like to acknowledge the clinical microbiology laboratory at the Aga Khan University Hospital for allowing us to conduct this research.

\section{Authors' contributions}

SR conceived the study, prepared the study protocol, involved in acquisition of data, performed review, and analyze the data and write-up of the manuscript. KJ and JF prepared the study protocol, interpret and analyzed the data and revised the manuscript. GJ, SC, AK, QK were involved in acquisition of data. All the authors read, approved the final manuscript and agreed to be accountable for all aspects of the work.

\section{Funding}

This study did not receive any fund.

\section{Availability of data and materials}

The datasets used and/or analysed during the current study are available from the corresponding author on reasonable request.
Ethics approval and consent to participate

The Aga Khan University ethics review committee ruled that no formal ethics approval was required for this study and waivered the need for consent as no additional intervention was done and samples sent for routine testing were used for the study (Reference \# 2019-1155-5751)

\section{Consent for publication}

Not applicable.

\section{Competing interests}

The authors declare that they have no competing interests.

Received: 18 May 2020 Accepted: 11 October 2020

Published online: 29 October 2020

\section{References}

1. Hashimoto S, Shime N. Evaluation of semi-quantitative scoring of Gram staining or semi-quantitative culture for the diagnosis of ventilatorassociated pneumonia: a retrospective comparison with quantitative culture. J Intensive Care. 2013;1(1):2.

2. Schurink CAM, Nieuwenhoven CAV, Jacobs JA, Rozenberg-Arska M, Joore HCA, Buskens E, et al. Clinical pulmonary infection score for ventilatorassociated pneumonia: accuracy and inter-observer variability. Intensive Care Med. 2004;30(2):217-24.

3. McCauley LM, Webb BJ, Sorensen J, Dean NC. Use of tracheal aspirate culture in newly intubated patients with community-onset pneumonia. Ann Am ThoracSoc. 2016;13(3):376-81. .

4. Grgurich PE, Hudcova J, Lei Y, Sarwar A, Craven DE. Diagnosis of ventilatorassociated pneumonia: controversies and working toward a gold standard. Curr Opinion Infect Dis. 2013;26(2):140-50.

5. Sanchez-Nieto J, Torres A, Garcia-Cordoba F, El-Ebiary M, Carrillo A, Ruiz J, et al. Impact of invasive and noninvasive quantitative culture sampling on outcome of ventilator-associated pneumonia: a pilot study. Am J RespirCrit Care Med. 1998;157(2):371-6. .

6. Wong LK, Barry AL, Horgan SM. Comparison of six different criteria for judging the acceptability of sputum specimens. J ClinMicrobiol. 1982;16(4):627-31.

7. Khawaja A, Zubairi AB, Durrani FK, Zafar A. Etiology and outcome of severe community acquired pneumonia in immunocompetent adults. BMC Infect Dis. 2013;13:94

8. Khan MS, Siddiqui SZ, Haider S, Zafar A, Zafar F, Khan RN, et al. Infection control education: impact on ventilator-associated pneumonia rates in a public sector intensive care unit in Pakistan. Trans R Soc Trop Med Hyg. 2009;103(8):807-11.

9. Kalil AC, Metersky ML, Klompas M, Muscedere J, Sweeney DA, Palmer LB, et al. Management of adults with hospital-acquired and ventilatorassociated pneumonia: 2016 clinical practice guidelines by the Infectious Diseases Society of America and the American Thoracic Society. Clin Infect Dis. 2016;63(5):e61-111.

10. Torres A, Niederman MS, Chastre J, Ewig S, Fernandez-Vandellos P, Hanberger $\mathrm{H}$, et al. International ERS/ESICM/ESCMID/ALAT guidelines for the management of hospital-acquired pneumonia and ventilator-associated pneumonia: guidelines for the management of hospital-acquired pneumonia (HAP)/ventilator-associated pneumonia (VAP) of the European 
Respiratory Society (ERS), European Society of Intensive Care Medicine (ESIC M), European Society of Clinical Microbiology and Infectious Diseases (ESCM ID) and AsociaciónLatinoamericana del Tórax (ALAT). EurRespir J. 2017;50(3): 1700582.

11. Fujitani S, Cohen-Melamed MH, Tuttle RP, Delgado E, Taira Y, Darby JM. Comparison of semi-quantitative endotracheal aspirates to quantitative non-bronchoscopicbronchoalveolar lavage in diagnosing ventilatorassociated pneumonia. Respiratory Care. 2009;54(11):1453-61.

12. Miller JM, Binnicker MJ, Campbell S, Carroll KC, Chapin KC, Gilligan PH, et al. A guide to utilization of the microbiology laboratory for diagnosis of infectious diseases: 2018 update by the Infectious Diseases Society of America and the American Society for Microbiology. Clin Infect Dis. 2018; 67(6): $\mathrm{e} 1-94$

13. McHugh ML. Interrater reliability: the kappa statistic. Biochemiamedica: Biochemiamedica. 2012;22(3):276-82.

14. Marquette CH, Copin M-C, Wallet F, Neviere R, Saulnier F, Mathieu D, et al. Diagnostic tests for pneumonia in ventilated patients: prospective evaluation of diagnostic accuracy using histology as a diagnostic gold standard. Am J RespirCrit Care Med. 1995;151(6):1878-88.

15. Torres A, el-Ebiary M, Padro L, Gonzalez J, De La Bellacasa JP, Ramirez J, et al. Validation of different techniques for the diagnosis of ventilatorassociated pneumonia Comparison with immediate postmortem pulmonary biopsy. Am J RespirCrit Care Med. 1994;149(2):324-31.

16. Fàbregas N, Ewig S, Torres A, El-Ebiary M, Ramirez J, de la Bellacasa JP, et al. Clinical diagnosis of ventilator associated pneumonia revisited: comparative validation using immediate post-mortem lung biopsies. Thorax. 1999;54(10): 867-73. .

17. Luna CM, Videla A, Mattera J, Vay C, Famiglietti A, Vujacich P, et al. Blood cultures have limited value in predicting severity of illness and as a diagnostic tool in ventilator-associated pneumonia. Chest. 1999;116(4):1075-84. .

18. Zubairi ABS, Zafar A, Salahuddin N, Haque AS, Waheed S, Khan J. Atypical pathogens causing community-acquired pneumonia in adults. JPMA J Pakistan Med Assoc. 2012;62(7):653.

19. Iqbal $N$, Irfan $M$, Siddique $F$, Arshad V, Zubairi ABS. Factors predicting inhospital mortality among patients admitted with community acquired pneumonia at a tertiary care hospital Karachi Pakistan. ClinRespir J. 2020 14(4):328-34.

20. Carugati M, Aliberti S, Sotgiu G, Blasi F, GoriMenendez AR, et al. Bacterial etiology of community-acquired pneumonia in immunocompetent hospitalized patients and appropriateness of empirical treatment recommendations: an international point-prevalence study. Eur J ClinMicrobiol Infect Dis. 2020;39:1513. .

\section{Publisher's Note}

Springer Nature remains neutral with regard to jurisdictional claims in published maps and institutional affiliations.

Ready to submit your research? Choose BMC and benefit from:

- fast, convenient online submission

- thorough peer review by experienced researchers in your field

- rapid publication on acceptance

- support for research data, including large and complex data types

- gold Open Access which fosters wider collaboration and increased citations

- maximum visibility for your research: over $100 \mathrm{M}$ website views per year

At $\mathrm{BMC}$, research is always in progress.

Learn more biomedcentral.com/submissions 